\title{
Sur le noyau sauvage des corps de nombres
}

\author{
par \\ JeAn-François Jaulent (Talence)
}

0. Introduction. Notre point de départ dans ce travail est une formule explicite particulièrement simple pour le symbole de Hilbert sur un corps local $K_{\mathfrak{p}}$ qui s'exprime comme suit : Soit $l$ un nombre premier tel que $K_{\mathfrak{p}}$ contienne les racines $2 l$-ièmes de l'unité pour un $r \geq 1$. Alors pour chaque racine $l^{r}$-ième de l'unité $\zeta$ contenue dans $K_{\mathfrak{p}}$ et tout $x$ de $K_{\mathfrak{p}}^{\times}$, le symbole de Hilbert correspondant est donné par la formule

$$
\left(\frac{\zeta, x}{\mathfrak{p}}\right)=\zeta^{\tilde{v}_{\mathfrak{p}}(x)},
$$

où $\widetilde{v}_{\mathfrak{p}}$ est une application à valeurs dans un $\mathbb{Z}_{l}$-réseau de $\mathbb{Q}_{l}$ définie à partir des logarithmes des valeurs absolues $l$-adiques.

Or l'intérêt de ce résultat n'est pas tant de donner un nouveau moyen de calcul des symboles de Hilbert (nous avons choisi d'en donner ici une preuve directe particulièrement simple, mais nous aurions pu aussi bien l'obtenir en transformant les formules explicites classiques qui font déjà intervenir le logarithme), que de relier naturellement les symboles sauvages à une application $\widetilde{v}_{\mathfrak{p}}$ qui n'est pas définie seulement modulo une congruence, mais prend effectivement ses valeurs dans un $\mathbb{Z}_{l}$-module de rang 1 , et peut ainsi être regardée comme l'analogue d'une valuation.

Plus précisement, si en chaque place non complexe $\mathfrak{p}$ d'un corps de nombres $K$ contenant les racines $2 l^{r}$-ièmes de l'unité, on convient de remplacer la valuation $\mathfrak{p}$-adique usuelle $v_{\mathfrak{p}}$ par celle logarithmique $\widetilde{v}_{\mathfrak{p}}$, on peut alors définir un groupe logarithmique de classes de diviseurs $\widetilde{\mathcal{C l}}_{K}$, analogue au l-groupe des classes au sens ordinaire $\mathcal{C} l_{K}$ (et donc d'une arithmétique voisine) et lié de surcroît au noyau symboles sauvages dans le groupe universel $K_{2}(K)$. L'isomorphisme obtenu

$$
l^{r} \mu_{K} \otimes_{\mathbb{Z}} \widetilde{\mathcal{C}}_{K} \simeq{ }^{r} H_{2}(K),
$$

déjà annoncé dans un article antérieur en termes idéliques (cf. [ $\left.\mathrm{J}_{2}\right]$, th. 2.12)

1991 Mathematics Subject Classification: 11R18, 11R70, 11S31. 
et reproduit sous une forme voisine quoique non canonique par d'autres auteurs (cf. [CK], prop. 2.1), prend toute son importance ici du fait que le groupe $\widetilde{\mathcal{C l}}_{K}$ n'est plus défini comme quotient du groupe des classes d'idèles, mais explicitement comme groupe de classes de diviseurs, donc algorithmiquement accessible aux méthodes numériques, ce qui ouvre la voie à une approche effective du groupe $H_{2}(K)$.

Nous commençons pour cela par rappeler quelques propriétés des valeurs absolues $l$-adiques et notamment leur interprétation en termes de corps de classes.

1. Rappel sur les valeurs absolues $l$-adiques. Si $K_{\mathfrak{p}}$ est un corps local, i.e. le complété en une place ultramétrique $\mathfrak{p}$ d'un corps de nombres $K$, la factorisation de son groupe multiplicatif

$$
K_{\mathfrak{p}}^{\times}=\mu_{\mathfrak{p}}^{0} U_{\mathfrak{p}}^{1} \pi_{\mathfrak{p}}^{\mathbb{Z}}
$$

fait intervenir le groupe $\mu_{\mathfrak{p}}^{0}$, d'ordre $N \mathfrak{p}-1$, formé des racines de l'unité d'ordre étranger à la caractéristique résiduelle $p$, le groupe $U_{\mathfrak{p}}^{1}=1+\mathfrak{p}$ des unités principales, et le groupe monogène multiplicatif engendré par une uniformisante arbitraire $\pi_{\mathfrak{p}}$.

Si maintenant $l$ est un nombre premier, la valeur absolue $l$-adique principale $|\cdot|_{\mathfrak{p}}$ est définie sur $K_{\mathfrak{p}}^{\times}$par la formule

$$
|x|_{\mathfrak{p}}= \begin{cases}\left\langle N \mathfrak{p}^{-v_{\mathfrak{p}}(x)}\right\rangle & \text { pour } \mathfrak{p} \nmid l \\ \left\langle N_{K_{\mathfrak{p}} / \mathbb{Q}_{l}}(x) N \mathfrak{p}^{-v_{\mathfrak{p}}(x)}\right\rangle & \text { pour } \mathfrak{p} \mid l,\end{cases}
$$

où $\langle\cdot\rangle$ est la projection canonique du groupe multiplicatif $\mathbb{Z}_{l}^{\times}$des unités de $\mathbb{Z}_{l}$ sur son sous-groupe libre maximal $1+2 l \mathbb{Z}_{l}$. Par composition avec le logarithme $l$-adique (qui est bien défini sur $1+2 l \mathbb{Z}_{l}$ par le développement en série $\left.\log (1+x)=\sum_{n \geq 1}(-1)^{n+1} x^{n} / n\right)$, on obtient ainsi un morphisme continu $\log ||_{\mathfrak{p}}$ sur le groupe topologique $K_{\mathfrak{p}}^{\times}$à valeurs dans le groupe additif $2 l \mathbb{Z}_{l}\left({ }^{1}\right)$.

Plus précisément, l'application obtenue induit canoniquement un $\mathbb{Z}_{l^{-}}$ morphisme, que nous continuerons à noter $\log ||_{\mathfrak{p}}$, du compactifié $l$-adique de $K_{\mathfrak{p}}^{\times}$, défini comme la limite projective

$$
\mathcal{K}_{\mathfrak{p}}^{\times}=\varliminf_{n} K_{\mathfrak{p}}^{\times} / K_{\mathfrak{p}}^{\times l^{n}},
$$

sur un sous-module d'indice fini de $\mathbb{Z}_{l}$ qui sera précisé plus loin. Il est commode d'écrire

$$
\mathcal{K}_{\mathfrak{p}}^{\times}=\mathcal{U}_{\mathfrak{p}} \cdot \pi_{\mathfrak{p}}^{\mathbb{Z}_{l}}
$$

$\left({ }^{1}\right)$ La définition ci-dessus de la valeur absolue l-adique principale diffère pour $l=2$ de celle utilisée dans $\left[J_{1}\right]$ où sont prises en compte les composantes sur le facteur $\{ \pm 1\}$ de $\mathbb{Z}_{2}^{\times}$. Cela est sans conséquence lorsqu'on compose par le logarithme 2-adique. 
comme produit direct de l'image $\mathcal{U}_{\mathfrak{p}}$ du groupe des unités de $K_{\mathfrak{p}}^{\times}$(qui s'identifie au $l$-sous-groupe de Sylow de $\mu_{\mathfrak{p}}^{0}$ pour $\mathfrak{p} \nmid l$, au groupe $U_{\mathfrak{p}}^{1}$ sinon) et du $\mathbb{Z}_{l}$-module libre de rang 1 engendré topologiquement par l'image de l'uniformisante $\pi_{\mathfrak{p}}$. Cela étant, l'application d'Artin identifie

- le compactifié $\mathcal{K}_{\mathfrak{p}}^{\times}$au groupe de $\operatorname{Galois} \operatorname{Gal}\left(K_{\mathfrak{p}}^{\mathrm{ab}} / K_{\mathfrak{p}}\right)$ de la pro-lextension abélienne maximale de $K_{\mathfrak{p}}$,

- le sous-groupe des unités $\mathcal{U}_{\mathfrak{p}}$ au sous-groupe d'inertie $\operatorname{Gal}\left(K_{\mathfrak{p}}^{\mathrm{ab}} / K_{\mathfrak{p}}^{\mathrm{nr}}\right)$ qui correspond à la $\mathbb{Z}_{l}$-extension non ramifiée $K_{\mathfrak{p}}^{\text {nr }}$ de $K_{\mathfrak{p}}$,

- et le noyau

$$
\mathcal{K}_{\mathfrak{p}}^{*}=\operatorname{Ker} \log ||_{\mathfrak{p}}
$$

au sous-groupe $\operatorname{Gal}\left(K_{\mathfrak{p}}^{\mathrm{ab}} / K_{\mathfrak{p}}^{\infty}\right)$ attaché à la $\mathbb{Z}_{l}$-extension cyclotomique $K_{\mathfrak{p}}^{\infty}$ de $K_{\mathfrak{p}}$ (cf. $\left[\mathrm{J}_{1}\right], \S 2$ ). En d'autres termes, $\mathcal{K}_{\mathfrak{p}}^{*}$ est le groupe des normes cyclotomiques dans $\mathcal{K}_{\mathfrak{p}}^{\times}$.

En particulier, on a $\mathcal{K}_{\mathfrak{p}}^{*}=\mathcal{U}_{\mathfrak{p}}$ pour $\mathfrak{p} \nmid l$.

2. Expression du symbole de Hilbert en termes de valeurs absolues. Notons $m_{\mathfrak{p}}$ l'ordre du sous-groupe de torsion $\mu_{\mathfrak{p}}$ de $K_{\mathfrak{p}}^{\times}$(i.e. le nombre de racines de l'unité contenues dans le corps local $K_{\mathfrak{p}}$ ), et $\omega_{\mathfrak{p}}$ l'application d'Artin attachée à $K_{\mathfrak{p}}$ (à valeurs dans le groupe de Galois de l'extension abélienne maximale de $K_{\mathfrak{p}}$ ). Avec ces notations le symbole de Hilbert est l'application bilinéaire de $K_{\mathfrak{p}}^{\times} \times K_{\mathfrak{p}}^{\times}$sur $\mu_{\mathfrak{p}}$ définie par l'identité

$$
\left(\frac{a, b}{K_{\mathfrak{p}}}\right)_{m_{\mathfrak{p}}}=\sqrt[m_{\mathfrak{p}}]{a}{\left(\omega_{\mathfrak{p}}(b)-1\right)}^{.}
$$

Cela posé, le résultat de départ de ce travail peut s'énoncer comme suit :

THÉORÈME 1 . Soient $l$ un nombre premier, $K_{\mathfrak{p}}$ un corps local contenant les racines $2 l^{r}$-ièmes de l'unité, et $m_{\mathfrak{p}}=2 l^{r} m_{\mathfrak{p}}^{\prime}$ (avec $\left.l \nmid m_{\mathfrak{p}}^{\prime}\right)$ l'ordre $d u$ groupe $\mu_{\mathfrak{p}}$. Alors, si $\zeta$ est une racine $l^{r}$-ième de l'unité dans $\mu_{\mathfrak{p}}$ et $x$ un élément quelconque de $K_{\mathfrak{p}}^{\times}$, le symbole de Hilbert correspondant est donné par la formule

$$
\left(\frac{\zeta, x}{K_{\mathfrak{p}}}\right)_{m_{\mathfrak{p}}}=\zeta^{-\log |x|_{\mathfrak{p}} / m_{\mathfrak{p}}}
$$

où ||$_{\mathfrak{p}}$ désigne la valeur absolue $l$-adique principale sur $K_{\mathfrak{p}}^{\times}$.

Démonstration. Ecrivons $m_{\mathfrak{p}}=(N \mathfrak{p}-1) p^{r_{\mathfrak{p}}}$ l'ordre de $\mu_{\mathfrak{p}}$, et distinguons deux cas suivant que la caractéristique résiduelle $p$ de $K_{\mathfrak{p}}$ est égale à $l$ ou non.

(i) Dans le cas modéré $l \neq p$, il est bien connu (cf. [Se], ch. XIV) que la

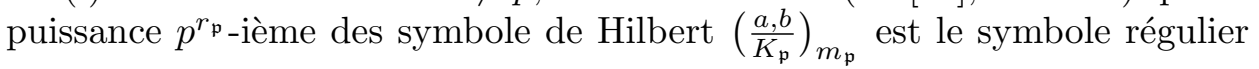


$(a, b)_{\mathfrak{p}}$ à valeurs dans $\mu_{\mathfrak{p}}^{0}$, caractérisé par la congruence

$$
\left(\frac{a, b}{K_{\mathfrak{p}}}\right)_{m_{\mathfrak{p}}}^{p^{r_{\mathfrak{p}}}}=(a, b)_{\mathfrak{p}} \equiv(-1)^{v_{\mathfrak{p}}(a) v_{\mathfrak{p}}(b)} \frac{a^{v_{\mathfrak{p}}(b)}}{b^{v_{\mathfrak{p}}(a)}}(\bmod \mathfrak{p}) .
$$

Il vient donc ici

$$
\left(\frac{\zeta, x}{K_{\mathfrak{p}}}\right)_{m_{\mathfrak{p}}}^{p^{r_{\mathfrak{p}}}}=\zeta^{v_{\mathfrak{p}}(x)}=\zeta^{-\log |x|_{\mathfrak{p}} / \log N \mathfrak{p}},
$$

compte tenu de l'expression dans ce cas de la valeur absolue $l$-adique $:|x|_{\mathfrak{p}}=$ $\left\langle N \mathfrak{p}^{-v_{\mathfrak{p}}(x)}\right\rangle$. D'autre part, puisque $K_{\mathfrak{p}}$ est supposé contenir les racines $2 l^{r}$ ièmes de l'unité, nous avons les congruences $N \mathfrak{p} \equiv 1\left(\bmod l^{r}\right)$ si $l$ est impair, et $N \mathfrak{p} \equiv 1\left(\bmod 2^{r+1}\right)$ si $l$ vaut 2 , ce qui nous donne dans les deux cas

$$
\frac{\log N \mathfrak{p}}{N \mathfrak{p}-1}=\frac{\log (1+(N \mathfrak{p}-1))}{N \mathfrak{p}-1} \equiv 1\left(\bmod l^{r}\right) \quad \text { dans } \mathbb{Z}_{l}^{\times} .
$$

Il vient donc finalement

$$
\left(\frac{\zeta, x}{K_{\mathfrak{p}}}\right)_{m_{\mathfrak{p}}}^{p_{\mathfrak{p}}}=\zeta^{-\log |x|_{\mathfrak{p}} /\left(N_{\mathfrak{p}}-1\right)},
$$

i.e.

$$
\left(\frac{\zeta, x}{K_{\mathfrak{p}}}\right)_{m_{\mathfrak{p}}}=\zeta^{-\log |x|_{\mathfrak{p}} /(N \mathfrak{p}-1) p^{r_{\mathfrak{p}}}}=\zeta^{-\log |x|_{\mathfrak{p}} / m_{\mathfrak{p}}},
$$

comme attendu.

(ii) Dans le cas sauvage $l=p$, considérons la quantité

$$
\left(\frac{\zeta, x}{K_{\mathfrak{p}}}\right)_{m_{\mathfrak{p}}}^{N \mathfrak{p}-1}=\sqrt[l_{\mathfrak{l}}]{\zeta}{ }^{\left(\omega_{\mathfrak{p}}(x)-1\right)} .
$$

Introduisons le sous-corps $C_{\mathfrak{l}}$ de $K_{\mathfrak{p}}$ engendré sur $\mathbb{Q}_{l}$ par les racines $l^{r_{\mathfrak{l}} \text {-ièmes }}$ de l'unité, et notons $\omega_{\mathfrak{l}}$ l'application d'Artin associée à $C_{l}$. Nous obtenons immédiatement

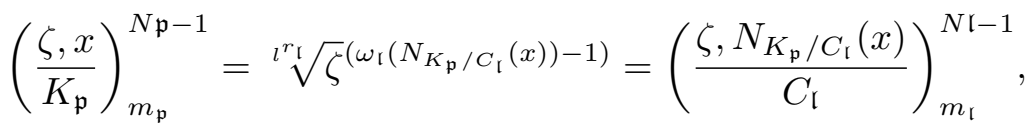

et nous sommes ainsi ramenés à un calcul dans le corps $C_{\mathfrak{l}}$, unique complété au-dessus de $l$ du corps cyclotomique $C$ engendré sur $\mathbb{Q}$ par les racines $l^{r_{1}}$ ièmes de l'unité. Or, ici, la formule du produit pour les symboles de Hilbert dans le corps $C$ nous permet d'écrire, pour tout $y$ de $C^{\times}$,

$$
\left(\frac{\zeta, y}{C_{\mathfrak{l}}}\right)_{m_{\mathfrak{l}}}^{m_{\mathfrak{l}} / m}=\prod_{\mathfrak{q} \neq \mathfrak{l}}\left(\frac{\zeta, y}{C_{\mathfrak{q}}}\right)_{m_{\mathfrak{q}}}^{-m_{\mathfrak{q}} / m}=\zeta^{-\Sigma_{\mathfrak{q} \neq \mathfrak{l}} \log |y|_{\mathfrak{q}} / m}
$$


avec

$$
\left\{\begin{array}{l}
m=2 l^{r}, \\
m_{\mathfrak{l}}=l^{r_{\mathfrak{r}}}(N \mathfrak{l}-1),
\end{array}\right.
$$

compte tenu des formules explicites déjà obtenues dans le cas modéré, c'està-dire :

$$
\left(\frac{\zeta, y}{C_{\mathfrak{l}}}\right)_{m_{\mathfrak{l}}}^{m_{\mathfrak{l}} / m}=\zeta^{-\log |y|_{\mathfrak{l}} / m}
$$

en vertu de la formule du produit pour les valeurs absolues, et finalement,

$$
\left(\frac{\zeta, y}{C_{\mathfrak{l}}}\right)_{m_{\mathfrak{l}}}^{N \mathfrak{l}-1}=\zeta^{-\log |y|_{\mathfrak{r}} / m}
$$

pour tout $y$ de $C^{\times}$donc, par densité, pour tout $y$ de $C_{\mathfrak{l}}^{\times}$; et en particulier,

$$
\left(\frac{\zeta, x}{K_{\mathfrak{p}}}\right)_{m_{\mathfrak{p}}}^{N_{\mathfrak{p}-1}}=\left(\frac{\zeta, N_{K_{\mathfrak{p}} / C_{\mathfrak{l}}}(x)}{C_{\mathfrak{l}}}\right)_{m_{\mathfrak{l}}}^{N \mathfrak{l}-1}=\zeta^{-\log |x|_{\mathfrak{p}} / l^{r_{\mathfrak{l}}}},
$$

ce qui nous donne donc, comme annoncé,

$$
\left(\frac{\zeta, x}{K_{\mathfrak{p}}}\right)=\zeta^{-\log |x|_{\mathfrak{p}} / m_{\mathfrak{p}}}
$$

Au passage, nous retrouverons la classique formule d'Artin-Hasse, généralisée pour $l$ impair par Iwasawa (cf. [Iw]), et qu'on peut aussi obtenir, indépendamment de toute considération de parité, via la théorie de Lubin-Tate (cf., par exemple, [La], Ch. 9).

SCOLIE. Conservons les notations du théorème, et supposons $l=2$. Alors, si $\xi$ est une racine $2^{r+1}$-ième primitive de l'unité dans $\mu_{\mathfrak{p}}$ et $x$ un élément quelconque de $K_{\mathfrak{p}}^{\times}$, il vient

$$
\left(\frac{\xi, x}{K_{\mathfrak{p}}}\right)_{m_{\mathfrak{p}}}=(-\xi)^{-\log |x|_{\mathfrak{p}} / m_{\mathfrak{p}}}
$$

Preuve. Pour $q \in 2^{r+1} \mathbb{Z}_{2}$, nous avons en effet

$$
\frac{\log (1+q)}{q} \equiv 1-\frac{q}{2}\left(\bmod 2^{r+1}\right)
$$

ce qui introduit un signe moins dans les calculs précédents en vertu de l'identité $\xi^{2^{r}}=-1$.

3. Valuations logarithmiques sur un corps de nombres. La formule explicite donnée au théorème 1 invite naturellement à prendre pour $l$-valuation logarithmique attachée à une place $\mathfrak{p}$ d'un corps de nombres $K$ contenant les racines $2 l^{r}$-ièmiques de l'unité la quantité $-\log |x|_{\mathfrak{p}} / m_{\mathfrak{p}}$. Malheureusement, cette définition donne lieu à des formules de transition inutilement compliquées lorsqu'on compare les $l$-valuations logarithmiques 
ainsi définies dans $K$ à celles qui leur correspondent dans une extension donné $L$ de $K$. C'est pourquoi il est préférable de procéder comme suit en posant :

DÉFinition. Soient $l$ un nombre premier, $K_{\mathfrak{p}}$ un corps local, et $m_{\mathfrak{p}}=$ $p^{r_{\mathfrak{p}}}(N \mathfrak{p}-1)$ le cardinal du sous-groupe de torsion $\mu_{\mathfrak{p}}$ de $K_{\mathfrak{p}}^{\times}$. Par l-symbole de Hilbert attaché à $K$, nous entendons le symbole sur $K_{\mathfrak{p}}$ à valeurs dans $\mu_{\mathfrak{p}}$ défini sur $K_{\mathfrak{p}}^{\times} \times K_{\mathfrak{p}}^{\times}$par

$$
\left(\frac{a, b}{\mathfrak{p}}\right)= \begin{cases}\left(\frac{a, b}{K_{\mathfrak{p}}}\right)_{m_{\mathfrak{p}}}^{p_{\mathfrak{p}}}=\left(\frac{a, b}{K_{\mathfrak{p}}}\right)_{N \mathfrak{p}-1} & \text { pour } \mathfrak{p} \nmid l, \\ \left(\frac{a, b}{K_{\mathfrak{p}}}\right)_{m_{\mathfrak{p}}}^{N \mathfrak{p}-1}=\left(\frac{a, b}{K_{\mathfrak{p}}}\right)_{p^{r_{\mathfrak{p}}}} & \text { pour } \mathfrak{p} \mid l,\end{cases}
$$

obtenu en prenant la partie régulière du symbole de Hilbert ordinaire pour les $\mathfrak{p}$ étrangers à $l$, et la partie sauvage pour les $\mathfrak{p}$ qui divisent $l$.

Cela étant, nous avons, en vertu du théorème 1 :

DÉFINITION \& THÉORÈME 3. Soient $l$ un nombre premier, et $K$ un corps de nombres contenant les racines $2 l^{r}$-ièmes de l'unité (pour un $r \geq 1$ ). Pour chaque place ultramétrique $\mathfrak{p}$ de $K$, nous appelons l-valuation logarithmique attachée à la place $\mathfrak{p}$ l'application $\widetilde{v}_{\mathfrak{p}}$ définie par $\left({ }^{2}\right)$ :

$$
\widetilde{v}_{\mathfrak{p}}=-\frac{\log ||_{\mathfrak{p}}}{\log N \mathfrak{p}}=v_{\mathfrak{p}} \quad \text { pour } \mathfrak{p} \nmid l \quad \text { et } \quad \widetilde{v}_{\mathfrak{p}}=-\frac{\log ||_{\mathfrak{p}}}{l^{r_{\mathfrak{p}}}} \quad \text { pour } \quad \mathfrak{p} \mid l,
$$

où $v_{\mathfrak{p}}$ est la $\mathfrak{p}$-valuation au sens ordinaire, $N \mathfrak{p}$ la norme absolue de la place considérée, $\log ||_{\mathfrak{p}}$ le logarithme de la valeur absolue l-adique principale associée, et l $l^{r_{\mathfrak{p}}}$ le nombre des racines de l'unité d'ordre l-primaire dans le complété $K_{\mathfrak{p}}$ pour $\mathfrak{p} \mid l$.

Avec ces conventions, pour toute racine $l^{r}$-ième de l'unité $\zeta$ dans $K$, les l-symboles de Hilbert attachés aux diverses places ultramétriques de $K$ satisfont l'identité

$$
\left(\frac{\zeta, x}{\mathfrak{p}}\right)=\zeta^{\tilde{v}_{\mathfrak{p}}(x)}, \quad \forall x \in K^{\times} .
$$

SCOLIE. Pour chaque place ultramétrique $\mathfrak{p}$, la l-valuation logarithmique $\widetilde{v}_{\mathfrak{p}}$ induit un $\mathbb{Z}_{l}$-épimorphisme (que nous continuerons à noter $\widetilde{v}_{\mathfrak{p}}$ ) du compactifiél-adique $\mathcal{K}_{\mathfrak{p}}^{\times}$de $K_{\mathfrak{p}}^{\times}$sur $\mathbb{Z}_{l}$, qui a pour noyau le groupe $\mathcal{K}_{\mathfrak{p}}^{*}$ des normes cyclotomiques.

$\left({ }^{2}\right)$ Pour quelques places de $K$ divisant $[K: \mathbb{Q}]$, la normalisation retenue ici pour la $l$-valuations logarithmique peut différer de celle donnée en toute généralité dans $\left[J_{3}\right]$. Comme on passe d'une normalisation à l'autre en multipliant par un élément de $\mathbb{Z}_{l}^{\times}$, c'est sans conséquence pour ce qui suit. 
Pr e u ve. Nous savons déjà que le noyau $\mathcal{K}_{\mathfrak{p}}^{*}=\operatorname{Ker} \log ||_{\mathfrak{p}}$ dans $\mathcal{K}_{\mathfrak{p}}^{\times}$est le groupe des normes cyclotomiques. Seule reste donc à vérifier la surjectivité de $\widetilde{v}_{\mathfrak{p}}$, laquelle résulte immédiatement du théorème 1 (et de son scolie pour $l=2$ ), puisque les symboles de Hilbert $\left(\frac{\zeta, x}{K_{\mathfrak{p}}}\right)_{m_{\mathfrak{p}}}$ pour $\zeta$ dans $\mu_{\mathfrak{p}}$ et $x$ dans $K_{\mathfrak{p}}^{\times}$prennent toutes les valeurs dans $\mu_{\mathfrak{p}}$.

Introduisons maintenant le $l$-adifié $\mathcal{J}_{K}$ du groupe des idèles de $K$, défini comme le produit

$$
\mathcal{J}_{K}=\prod_{\mathfrak{p}}^{\text {res }} \mathcal{K}_{\mathfrak{p}}^{\times}
$$

des compactifiés $l$-adiques $\mathcal{K}_{\mathfrak{p}}^{\times}$restreint aux familles $\left(x_{\mathfrak{p}}\right)_{\mathfrak{p}}$ qui vérifient $x_{\mathfrak{p}} \in$ $\mathcal{U}_{\mathfrak{p}}$ pour presque tout $\mathfrak{p}$. Puisque $\mathcal{K}_{\mathfrak{p}}^{*}$ coïncide avec $\mathcal{U}_{\mathfrak{p}}$ pour $\mathfrak{p} \nmid l$, la famille $\widetilde{v}=\left(v_{\mathfrak{p}}\right)_{\mathfrak{p}}$ des $l$-valuations logarithmiques envoie $\mathcal{J}_{K}$ sur la somme directe $\bigoplus_{\mathfrak{p}} \mathbb{Z}_{l} \mathfrak{p}$ d'autant d'exemplaires de $\mathbb{Z}_{l}$ que de places ultramétriques de $K$. Plus précisement :

DÉfinition \& Proposition 4. Convenons d'appeler l-diviseur logarithmique toute somme formelle finie

$$
\mathfrak{d}=\sum_{\mathfrak{p}} a_{\mathfrak{p}} \mathfrak{p}
$$

de places ultramétriques de $K$ à coefficients dans $\mathbb{Z}_{l}$. Cela posé, la famille $\widetilde{v}=\left(\widetilde{v}_{\mathfrak{p}}\right)_{\mathfrak{p}}$ des l-valuations logarithmiques définit un $\mathbb{Z}_{l}$-épimorphisme continu du l-adifié $\mathcal{J}_{K}=\prod_{\mathfrak{p}}^{\text {res }} \mathcal{K}_{\mathfrak{p}}^{\times}$du groupe des idèles de $K$ sur le groupe $\mathcal{D} l_{K}=\bigoplus_{\mathfrak{p}} \mathbb{Z}_{l} \mathfrak{p}$ des l-diviseurs logarithmiques qui a pour noyau le groupe $\mathcal{J}_{K}^{*}=\prod_{\mathfrak{p}} \mathcal{K}_{\mathfrak{p}}^{*}$ des normes cyclotomiques locales.

Preuve. C'est clair $\left({ }^{3}\right)$.

Désignons enfin par $\mathcal{R}_{K}=\mathbb{Z}_{l} \otimes_{\mathbb{Z}} K^{\times}$le sous-groupe principal de $\mathcal{J}_{K}$, de sorte que le quotient $\mathcal{C} l_{K}=\mathcal{J}_{K} / \mathcal{R}_{K}$ s'identifie, par la théorie $l$-adique du corps de classes, au groupe de $\operatorname{Galois} \operatorname{Gal}\left(K^{\mathrm{ab}} / K\right)$ de la pro-l-extension abélienne maximale de $K$ (cf. $\left[\mathrm{J}_{1}\right], \S 1$ ).

La formule du produit pour les valeurs absolues montre que $\mathcal{R}_{K}$ est contenu dans le noyau

$$
\widetilde{\mathcal{J}}_{K}=\left\{\left.\left(x_{\mathfrak{p}}\right)_{\mathfrak{p}} \in \mathcal{J}_{K}\left|\prod_{\mathfrak{p}}\right| x_{\mathfrak{p}}\right|_{\mathfrak{p}}=1\right\}
$$

qui s'interprète comme le sous-groupe des normes cyclotomiques globales dans $\mathcal{J}_{K}\left(\right.$ cf. $\left.\left[\mathrm{J}_{1}\right], \S 3\right)$.

$\left({ }^{3}\right)$ La définition ci-dessus précise celle donnée dans $\left[\mathrm{J}_{2}\right]$ : c'est évidemment la formule explicite du théorème 1 qui justifie la normalisation adoptée ici. 
En particulier, transportant cette inclusion par la valuation $\widetilde{v}$, nous obtenons :

Définition \& Proposition 5. Convenons de définir le degré d'un ldiviseur logarithmique $\mathfrak{d}$ par la formule

$$
\operatorname{deg}\left(\sum_{\mathfrak{p}} a_{\mathfrak{p}} \mathfrak{p}\right)=\sum_{\mathfrak{p}} a_{\mathfrak{p}} \operatorname{deg} \mathfrak{p}, \quad \text { avec } \quad \operatorname{deg} \mathfrak{p}= \begin{cases}\log N \mathfrak{p} & \text { pour } \mathfrak{p} \nmid l, \\ l^{r_{\mathfrak{p}}} & \text { pour } \mathfrak{p} \mid l,\end{cases}
$$

en affectant à chaque place ultramétrique $\mathfrak{p}$ un poids égal au logarithme de la norme absolue de $\mathfrak{p}$ pour $\mathfrak{p} \nmid l$ et au nombre $l^{r_{\mathfrak{p}}}$ de racines sauvages de l'unité dans $K_{\mathfrak{p}}$ pour $\mathfrak{p} \mid l$. Cela étant, l'application $\widetilde{\text { div }}$ induite par les l-valuations logarithmiques $\widetilde{v}=\left(\widetilde{v}_{\mathfrak{p}}\right)_{\mathfrak{p}}$ envoie le sous-groupe principal $\mathcal{R}_{K}=\mathbb{Z}_{l} \otimes_{\mathbb{Z}} K^{\times}$ de $\mathcal{J}_{K}$ dans le groupe $\widetilde{\mathcal{D l}}_{K}$ des l-diviseurs logarithmiques de degré nul.

Nous disons que l'image $\widetilde{\mathcal{P}} l_{K}=\widetilde{\operatorname{div}}\left(R_{K}\right)$ est le sous-groupe principal de $\widetilde{\mathcal{D l}}_{K}$, et que le quotient $\widetilde{\mathcal{C l}}_{K}=\widetilde{\mathcal{D}}_{K} / \widetilde{\mathcal{P l}}_{K}$ est le l-groupe des classes logarithmiques de $K$.

4. Application au noyau sauvage de $K_{2}(K)$. Conservons les notations précédentes, et intéressons nous au $l$-sous-groupe de Sylow du noyau $H_{2}(K)$ dans $K_{2}(K)$ des symboles sauvages ou de Hilbert. Le théorème de Moore sur le $K_{2}$ des corps de nombres nous donne une suite exacte courte

$$
1 \rightarrow H_{2}(K) \rightarrow K_{2}(K) \stackrel{\widetilde{\lambda}}{\rightarrow} \underset{\mathfrak{p}}{\widetilde{p}} \mu_{\mathfrak{p}} \rightarrow 1,
$$

où l'application $\widetilde{\lambda}$, induite par les symboles de Hilbert, prend ses valeurs dans le sous-groupe $\widetilde{\bigoplus}_{\mathfrak{p}} \mu_{\mathfrak{p}}$ de la somme directe des groupes locaux de racines de l'unité attachés aux places non complexes de $K$, formé des familles $\left(\zeta_{\mathfrak{p}}\right)_{\mathfrak{p}}$ qui satisfont la formule du produit

$$
\prod_{\mathfrak{p}} \zeta_{\mathfrak{p}}^{m_{\mathfrak{p}} / m}=1
$$

où $m_{\mathfrak{p}}$ désigne l'ordre de $\mu_{\mathfrak{p}}$ et $m$ celui du sous-groupe de torsion $\mu_{K}$ de $K^{\times}$.

Supposons donc que $K$ contienne les racines $2 l^{r}$-ièmes de l'unité, écrivons $m=2 l^{r} m^{\prime}$ (avec $r \geq 1$ et $l \nmid m^{\prime}$ ) l'ordre de $\mu_{K}$, et formons la suite exacte du serpent associée au diagramme commutatif

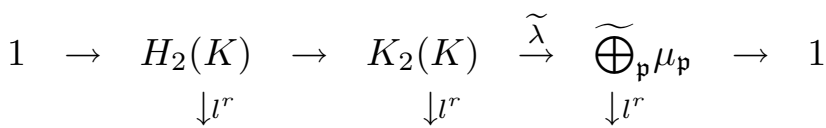

$$
\begin{aligned}
& 1 \rightarrow H_{2}(K) \rightarrow K_{2}(K) \stackrel{\widetilde{\lambda}}{\rightarrow} \widetilde{\bigoplus}_{\mathfrak{p}} \mu_{\mathfrak{p}} \rightarrow 1
\end{aligned}
$$

Dans la suite exacte longue obtenue

$$
1 \rightarrow l^{r} H_{2}(K) \rightarrow l^{r} K_{2}(K) \stackrel{\widetilde{\lambda}}{\rightarrow} \widetilde{\bigoplus}_{l^{r}} \mu_{\mathfrak{p}} \rightarrow{ }^{l_{r}} H_{2}(K) \rightarrow{ }^{l^{r}} K_{2}(K) \stackrel{\widetilde{\lambda}}{\rightarrow} \widetilde{\oplus} l^{r} \mu_{\mathfrak{p}} \rightarrow 1,
$$


les deux groupes à droite sont isomorphes d'après un résultat de Tate $K_{2}(K) / K_{2}(K)^{l^{r}} \simeq \widetilde{\bigoplus} \mu_{\mathfrak{p}} / \mu_{\mathfrak{p}}^{l^{r}}$ (cf. [Ta]); et les éléments d'ordre divisant $l^{r}$ dans $K_{2}(K)$ sont de la forme $\{\zeta, x\}$ pour $\zeta \in l^{r} \mu_{K}$ et $x$ dans $K^{\times}$, c'est-à-dire qu'il proviennent via le symbole universel $\{\cdot, \cdot\} \mid K^{\times} \times K^{\times} \rightarrow K_{2}(K)$ du produit tensoriel ${ }_{l^{r}} \mu_{K} \otimes_{\mathbb{Z}} K^{\times} \simeq{ }_{l^{r}} \mu_{K} \otimes_{\mathbb{Z}_{l}} \mathcal{R}_{K}$. Or :

Lemme 6. L'application $\zeta \otimes\left(\sum_{\mathfrak{p}} \nu_{\mathfrak{p}} \mathfrak{p}\right) \mapsto\left(\zeta^{\nu_{\mathfrak{p}}}\right)_{\mathfrak{p}}$ est un isomorphisme $d u$ tensorisé $l^{r} \mu_{K} \otimes \widetilde{\mathcal{D}}_{K}$ sur la somme directe restreinte $\widetilde{\bigoplus} l^{r} \mu_{\mathfrak{p}}$.

Preuve. Fixons une racine primitive $l^{r}$-ième de l'unité $\zeta$ dans $K$, et considérons un élément $\left(\zeta^{\nu_{\mathfrak{p}}}\right)_{\mathfrak{p}}$ de la somme directe $\bigoplus_{\mathfrak{p}} l^{r} \mu_{\mathfrak{p}}$ satisfaisant la formule du produit, i.e. vérifiant la congruence

$$
\sum_{\mathfrak{p}} \nu_{\mathfrak{p}} \frac{m_{\mathfrak{p}}}{m} \equiv 0\left(\bmod l^{r}\right) .
$$

Les $\mathfrak{p}$ pour lesquels $\nu_{\mathfrak{p}}$ est non nul étant en nombre fini, le théorème de Chebotarev nous garantit l'existence d'une place $\mathfrak{q}$ en dehors du support de la famille $\left(\nu_{\mathfrak{p}}\right)_{\mathfrak{p}}$ qui ne se décompose pas dans l'extension abélienne $K\left[\zeta_{2 l^{r+1}}\right] / K$, c'est-à-dire pour laquelle $m_{\mathfrak{q}} / m$ est inversible dans $\mathbb{Z}_{l}$. La quantité

$$
\nu_{\mathfrak{q}}=-\frac{m}{m_{\mathfrak{q}}} \sum_{\mathfrak{p}} \nu_{\mathfrak{p}} \frac{m_{\mathfrak{p}}}{m}
$$

est donc dans $l^{r} \mathbb{Z}_{l}$, et si $\mathfrak{d}$ est le diviseur logarithmique de degré nul défini par $\mathfrak{d}=\sum_{\mathfrak{p}} \nu_{\mathfrak{p}} \mathfrak{p}+\nu_{\mathfrak{q}} \mathfrak{q}$, il est clair que $\zeta \otimes \mathfrak{d}$ est un antécédent de la famille $\left(\zeta^{\nu_{\mathfrak{p}}}\right)_{\mathfrak{p}}$; ce qui établit la surjectivité. L'injectivité est, elle, immédiate.

Récapitulant ce qui précède, nous obtenons le diagramme exact :

$$
\begin{array}{cccccc}
l^{r} K_{2}(K) & \stackrel{\widetilde{\lambda}}{\rightarrow} & \underset{\uparrow}{\bigoplus_{\mathfrak{p}} l^{r} \mu_{\mathfrak{p}}} & \rightarrow & l^{r} H_{2}(K) & \rightarrow 1 \\
l^{r} \mu_{K} \otimes \mathcal{R}_{K} & \stackrel{\widetilde{v}}{\rightarrow} & l^{r} \mu_{K} \otimes \widetilde{\mathcal{D}}_{K} & \rightarrow & l^{r} \mu_{K} \otimes \widetilde{\mathcal{C l}}_{K} & \rightarrow 1
\end{array}
$$

et le carré à gauche est commutatif en vertu du théorème 1 . Il vient donc finalement, comme annoncé dans $\left[\mathrm{J}_{1}\right]$ (th. 2.12) :

ThÉORÈme 7. Soient $l$ un nombre premier et $K$ un corps de nombres contenant les racines $2 l^{r}$-ièmes de l'unité pour un $r \geq 1$. Ecrivons $H_{2}(K)$ le noyau dans $K_{2}(K)$ des symboles de Hilbert, ${ }_{l^{r}} \mu_{K}$ le sous-groupe de $l^{r}$-torsion de $K^{\times}$, et $\widetilde{\mathcal{C l}}_{K}$ le l-groupe des classes logarithmiques de $K$. Les l-valuations logarithmiques induisent alors un isomorphisme canonique

$$
H_{2}(K) / H_{2}(K)^{l^{r}} \simeq l^{r} \mu_{K} \otimes \widetilde{\mathcal{C}}_{K} .
$$

En particulier, le l-sous-groupe de Sylow du noyau hilbertien et le l-groupe des classes logarithmiques sont simultanément triviaux. 
SCOLIE. Le même résultat $H_{2}(K) / H_{2}(K)^{2^{r+1}} \simeq{ }_{2^{r+1}} \mu_{K} \otimes \widetilde{\mathcal{C l}}_{K}$ vaut encore à l'ordre $r+1$ pour $l=2$, mais dans ce cas l'isomorphisme ${ }_{2^{r+1} \mu_{K}} \otimes \widetilde{\mathcal{D l}}_{K} \simeq$ $\widetilde{\bigoplus}_{\mathfrak{p} 2^{r+1}} \mu_{\mathfrak{p}}$ est donné, pour $\xi$ racine primitive $2^{r+1}$-ième de l'unité, par

$$
\xi \otimes\left(\sum \nu_{\mathfrak{p} p}\right) \mapsto\left(\left(-\xi^{\nu_{\mathfrak{p}}}\right)_{\mathfrak{p} \mid 2},\left(\xi^{\nu_{\mathfrak{p}}}\right)_{\mathfrak{p} \nmid 2}\right) .
$$

L'isomorphisme obtenu est à rapprocher de celui donné par Keune (cf. $[\mathrm{Ke}]$, th. 6.6) qui fait intervenir les $l$-groupes de $l$-classes au sens habituel $\mathcal{C} l_{K}^{\prime}$, mais impose en revanche de monter dans la tour cyclotomique, alors que le théorème 7 ci-dessus se lit directement dans le corps $K$. Une version voisine a également été établie par Kramer et Candiotti (cf. [CK], prop. 2.1), mais l'isomorphisme qu'ils donnent n'est pas canonique et en particulier il ne respecte pas l'action galoisienne. Bien entendu, le point essentiel ici est la commutativité du diagramme c'est-à-dire le fait que l'application $\widetilde{\lambda}$ provient de la valuation logarithmique $\widetilde{v}$.

Il est d'autre part tentant, compte tenu de l'isomorphisme entre quotients d'exposant $l^{r}$ donné par le théorème, d'essayer de construire directement un morphisme canonique non plus entre quotients mais entre sous-groupes d'exposant $l^{r}$. Il se trouve que c'est en effet possible, du moins sous la conjecture de Gross, mais ici encore en acceptant de monter dans la tour cyclotomique $K_{\infty} / K$.

5. Extension aux corps surcirculaires. Supposons donc toujours que $K$ soit un corps de nombres contenant les racines $2 l^{r}$-ièmes de l'unité, et introduisons sa $\mathbb{Z}_{l}$-extension cyclotomique $K_{\infty}=\bigcup_{n \in \mathbb{N}} K_{n}$, avec, disons $\left({ }^{4}\right)$,

$$
K_{n}=K\left[\zeta_{l^{n}}\right],
$$

pour un système cohérent $\left(\zeta_{l^{n}}\right)_{n \in \mathbb{N}}$ de racines primitives $l^{n}$-ièmes de l'unité (i.e. vérifiant $\zeta_{l^{n+1}}^{l}=\zeta_{l^{n}}$ ).

Si maintenant $\mathfrak{p}$ est une place ultramétrique de $K, \mathfrak{p}_{n}$ une place de $K_{n}$ au-dessus de $\mathfrak{p}$ et $K_{\mathfrak{p}_{n}}$ le complété de $K_{n}$ correspondant, la définition de la valeur absolue $l$-adique montre que l'on a $|x|_{\mathfrak{p}_{n}}=|x|_{\mathfrak{p}}^{\left[K_{\mathfrak{p}_{n}}: K_{\mathfrak{p}}\right]}$ et donc

$$
\widetilde{v}_{\mathfrak{p}_{n}}(x)=\widetilde{v}_{\mathfrak{p}}(x) \text { pour tout } x \text { de } K^{\times},
$$

c'est-à-dire que la valuation logarithmique d'un élément $x$ de $K^{\times}$en une place $\mathfrak{p}$ peut se calculer en n'importe quelle place au-dessus de $\mathfrak{p}$ à chaque étage fini de la tour d'extensions $K_{\infty} / K$. Il est ainsi naturel de regarder $\mathcal{D} l_{K}$ comme un sous-module de $\mathcal{D} l_{K_{n}}$ en écrivant formellement chaque place

$\left({ }^{4}\right) K_{\infty}$ est ce qu'il est convenu d'appeler un corps surcirculaire (ou un $\mathbb{Z}_{l}$-corps dans la terminologie d'Iwasawa). 
ultramétrique $\mathfrak{p}$ de $K$ comme somme des places de $K_{n}$ qui sont au-dessus :

$$
\mathfrak{p}=\sum_{\mathfrak{p}_{n} \mid \mathfrak{p}} \mathfrak{p}_{n}
$$

L'identité immédiate $\operatorname{deg}_{K_{n}}\left(\sum_{\mathfrak{p}_{n} \mid \mathfrak{p}} \mathfrak{p}_{n}\right)=\left[K_{n}: K\right] \operatorname{deg}_{K}(\mathfrak{p})$ montre alors que l'on a

$$
\operatorname{deg}_{K_{n}}(\mathfrak{d})=\left[K_{n}: K\right] \operatorname{deg}_{K}(\mathfrak{d})
$$

pour tout diviseur logarithmique $\mathfrak{d}$ de $K$, et qu'en particulier $\widetilde{\mathcal{D l}}_{K}$ est contenu dans $\widetilde{\mathcal{D l}}_{K_{n}}$.

Plus généralement, puisque chaque place ultramétrique $\mathfrak{p}$ de $K$ est finiment décomposée dans la tour cyclotomique $K_{\infty} / K$, nous pouvons encore écrire à la limite

$$
\mathfrak{p}=\sum_{\mathfrak{p}_{\infty} \mid \mathfrak{p}} \mathfrak{p}_{\infty}
$$

comme somme des places $K_{\infty}$ qui sont au-dessus, avec pour tout $x$ de $K^{\times}$, $\widetilde{v}_{\mathfrak{p}_{\infty}}(x)=\widetilde{v}_{\mathfrak{p}}(x)$, de sorte que l'ensemble de cette discussion peut se résumer comme suit :

Proposition 8. Pour chaque place ultramétrique $\mathfrak{p}_{\infty}$ du corps surcirculaire $K_{\infty}=\bigcup_{n \in \mathbb{N}} K_{n}$, définissons la l-valuation logarithmique $\widetilde{v}_{\mathfrak{p}_{\infty}}$ sur le groupe

$$
\mathcal{R}_{K_{\infty}}=\mathbb{Z}_{l} \otimes_{\mathbb{Z}} K_{\infty}^{\times}=\bigcup_{n \in \mathbb{N}}\left(\mathbb{Z}_{l} \otimes_{\mathbb{Z}} K_{n}^{\times}\right)
$$

en posant pour tout $x$ de $\mathcal{R}_{K_{\infty}}$ et tout $n$ assez grand pour que $x$ soit dans $\mathcal{R}_{K_{n}}$,

$$
\widetilde{v}_{\mathfrak{p}_{\infty}}(x)=\widetilde{v}_{\mathfrak{p}_{n}}(x) .
$$

Regardons enfin $\widetilde{\mathcal{D l}}_{K}$ comme un sous-module de $\widetilde{\mathcal{D}}_{K_{\infty}}=\bigcup_{n \in \mathbb{N}} \widetilde{\mathcal{D l}}_{K_{n}}$ en définissant les morphismes d'inclusion par les identités valables pour chaque place finie $\mathfrak{p}_{n}$ de $K_{n}$ :

$$
\mathfrak{p}_{n}=\sum_{\mathfrak{p}_{\infty} \mid \mathfrak{p}_{n}} \mathfrak{p}_{\infty}
$$

Nous obtenons alors le diagramme commutatif:

$$
\begin{array}{ccccc}
\widetilde{\mathcal{D} l} & =\widetilde{\bigoplus}_{\mathfrak{p}} \mathbb{Z}_{l} \mathfrak{p} \\
\uparrow \widetilde{\mathcal{D} l} K_{K_{n}}=\widetilde{\bigoplus}_{\mathfrak{p}_{n}} \mathbb{Z}_{l} \mathfrak{p}_{n} & \rightarrow & \widetilde{\mathcal{D} l_{K_{\infty}}}=\widetilde{\bigoplus}_{\mathfrak{p}_{\infty}} \mathbb{Z}_{l} \mathfrak{p}_{\infty} \\
\mathcal{R}_{K}=\mathbb{Z}_{l} \otimes_{\mathbb{Z}} K^{\times} & \rightarrow & \mathcal{R}_{K_{n}}=\mathbb{Z}_{l} \otimes_{\mathbb{Z}} K_{n}^{\times} & \rightarrow & \mathcal{R}_{K_{\infty}}=\mathbb{Z}_{l} \otimes_{\mathbb{Z}} K_{\infty}^{\times}
\end{array}
$$

où les flèches horizontales sont les inclusions canoniques et celles verticales sont induites par les l-valuations logarithmiques.

DÉFInITION 9. Nous disons que le quotient $\widetilde{\mathcal{C l}}_{K_{\infty}}=\widetilde{\mathcal{D}}_{K_{\infty}} / \widetilde{\mathcal{P l}}_{K_{\infty}}=$ $\underline{\mathrm{lim}_{\mathrm{C} l}} \widetilde{\mathrm{Cl}}_{K_{n}}$ du groupe des diviseurs de degré nul $\widetilde{\mathcal{D} l} K_{\infty}$ par son sous-groupe 
principal $\widetilde{\mathcal{P}} K_{K_{\infty}}=\widetilde{\operatorname{div}}\left(\mathcal{R}_{K_{\infty}}\right)$ est le l-groupe des classes logarithmiques $\mathrm{du}$ corps $K_{\infty}$.

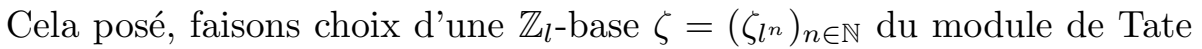
$\mathbb{T}_{l}=\varliminf \mu_{l^{n}}$ (c'est-à-dire, d'un système projectif de racines primitives $l^{n}$ ièmes de l'unité, disons $\left(\zeta_{l^{n}}\right)$ avec $\zeta_{l^{n+1}}^{l}=\zeta_{l^{n}}$, pour tout $n \in \mathbb{N}$ ). Avec les conventions ci-dessus, le théorème 7 s'énonce à la limite comme suit :

THÉORÈme 10. Soient $l$ un nombre premier, $K$ un corps de nombres contenant les racines $2 l^{r}$-ièmes de l'unité, et $K_{\infty}$ la $\mathbb{Z}_{l}$-extension cyclotomique de K. L'application

$$
\left\{\zeta_{l^{r}}, x\right\} \mapsto \zeta \otimes \widetilde{c l}_{K_{\infty}}\left(l^{-r} \widetilde{\operatorname{div}}(x)\right),
$$

où $\widetilde{c l}_{K_{\infty}}$ désigne la surjection canonique de $\widetilde{\mathcal{D l}}_{K_{\infty}}$ sur $\widetilde{\mathcal{C l}}_{K_{\infty}}$, est un épimorphisme du l-groupe de Sylow $l_{\infty} H_{2}\left(K_{\infty}\right)$ du noyau sauvage $H_{2}\left(K_{\infty}\right)$ dans $K_{2}\left(K_{\infty}\right)$, sur le tensorisé $\mathbb{T}_{l} \otimes_{\mathbb{Z}_{l}} \widetilde{\mathcal{C}} K_{\infty}^{\text {tor }}$ du sous-groupe de torsion du lgroupe des classes logarithmiques de $K_{\infty}$, qui a pour noyau le sous-groupe de $l_{\infty} \mathrm{H}_{2}\left(K_{\infty}\right)$ formé des symboles $\{\zeta, x\}$ construits sur les normes cyclotomiques.

En particulier, sous la conjecture de Gross dans $K_{\infty}$ on obtient ainsi l'isomorphisme

$$
l^{\infty} H_{2}\left(K_{\infty}\right) \simeq \mathbb{T}_{l} \otimes_{\mathbb{Z}_{l}} \widetilde{\mathcal{C}} l_{K_{\infty}} .
$$

Remarques. (i) Ce dernier résultat est sans rapport immédiat avec celui énoncé à la fin de la section précédente : On peut aussi, bien entendu, passer à la limite dans l'isomorphisme entre quotients donné par le théorème 7. Il convient cependant pour cela de remplacer le système inductif associé aux applications d'extension par le système projectif associé aux applications normes ou à leurs équivalents (i.e. au transfert en termes de $K$-théorie). L'isomorphisme final entre modules d'Iwasawa (qui est, lui, indépendant de la conjecture de Gross) :

$$
\varliminf_{l} H_{2}\left(K_{n}\right) \simeq \mathbb{T}_{l} \otimes_{\mathbb{Z}_{l}} \varliminf \widetilde{\mathcal{C l}}_{K_{n}}
$$

est essentiellement bien connu (cf. $\left.\left[\mathrm{J}_{2}\right]\right)$. Par la théorie du corps de classes, la limite projective $\varliminf \widetilde{\mathcal{C l}}_{K_{n}}$ s'interprète en effet comme le groupe de Galois $\operatorname{Gal}\left(C_{\infty}^{\prime} / K_{\infty}\right)$ attaché à la pro-l-extension abélienne maximale de $K_{\infty}$ qui est complètement décomposée en chacune de ses places. On notera dans ce cas que la condition normique sur le système projectif de racines de l'unité s'écrit pour $l=2: N_{K_{n+1} / K_{n}}\left(\zeta_{2^{n+1}}\right)=\zeta_{2^{n}}$, i.e. $\zeta_{2^{n+1}}^{2}=-\zeta_{2^{n}}$, ce qui introduit bien le signe moins attendu ici, conformément au scolie du théorème 7.

(ii) L'isomorphisme obtenu est à rapprocher, en revanche, du théorème principal de l'article de Kolster (cf. [Ko], th. 2.7). On notera cependant que la définition des groupes $\widetilde{\mathcal{C}}_{K_{n}}$ est strictement de type corps de classes, tandis 
que celle des groupes $\mathfrak{H}_{K_{n}} / \mathfrak{N}_{K_{n}}$ (dans les notations de [ $\left.\mathrm{J}_{2}\right]$ ) intervenant dans [Ko] relève de la théorie de Kummer. L'isomorphisme canonique résultant (sous Gross) $\widetilde{\mathcal{C l}}_{K_{\infty}} \simeq \mathfrak{H}_{K_{\infty}} / \mathfrak{N}_{K_{\infty}}$ peut être regardé comme l'une des formes les plus élaborées du Spiegelungssatz de Leopoldt.

Preuve du théorème 10. Les résultats cohomologiques de Tate (cf. [Ta]) montrent que les éléments de $K_{2}\left(K_{\infty}\right)$ d'ordre d'une puissance de $l$ sont ceux de la forme $\left\{\zeta_{l^{r}}, x\right\}$, avec $r \geq 1$ et $x \in K_{\infty}^{\times}$(que nous pouvons tout aussi bien regarder dans $\mathcal{R}_{K_{\infty}}=\mathbb{Z}_{l} \otimes_{\mathbb{Z}} K_{\infty}^{\times}$, puisqu'il n'est défini qu'à une puissance $l^{r}$-ième près). Or, ici, pour $\left\{\zeta_{l^{r}}, x\right\} \in H_{2}\left(K_{\infty}\right)$, l'expression explicite des $l$-symboles de Hilbert donnée par le théorème 3 implique que le diviseur logarithmique $\widetilde{\operatorname{div}}(x)$ tombe dans le sous-module $l^{r} \widetilde{\mathcal{D}} l_{K_{\infty}}$ de $\widetilde{\mathcal{D} l_{K_{\infty}}}$, ce qui invite à considérer la classe $\widetilde{c l}_{K_{\infty}}\left(l^{-r} \widetilde{\operatorname{div}}(x)\right)$ dans le groupe $\widetilde{\mathcal{C}} K_{K_{\infty}}$.

Cela étant, l'application $\psi \mid \zeta_{l^{r}} \otimes x \mapsto \zeta \otimes \widetilde{c l}_{K_{\infty}}\left(l^{-r} \widetilde{\operatorname{div}}(x)\right)$ est clairement

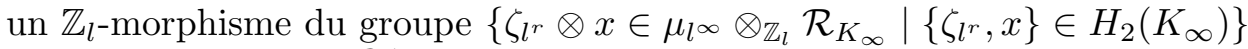
sur le produit $\mathbb{T}_{l} \otimes_{\mathbb{Z}_{l}} \widetilde{\mathcal{C} l} K_{\infty}^{\text {tor }}$. Son noyau est formé des éléments $\zeta_{l^{r}} \otimes x$ pour lesquels $\widetilde{\operatorname{div}}(x)$ est de la forme $l^{r} \widetilde{\operatorname{div}}(y)$ pour un $y$ de $\mathcal{R}_{K_{\infty}}$, autrement dit pour lesquels $x$ s'écrit $x=\varepsilon y^{l^{r}}$ avec $\widetilde{\operatorname{div}}(\varepsilon)=0$. Il vient donc

$$
\operatorname{Ker} \psi=\left\{\zeta_{l^{r}} \otimes \varepsilon \in \mu_{l^{\infty}} \otimes_{\mathbb{Z}_{l}} \mathcal{R}_{K_{\infty}} \mid \widetilde{\operatorname{div}}(\varepsilon)=0\right\} .
$$

Et le noyau de $\psi$ est le sous-module de $\mu_{l^{\infty}} \otimes \mathcal{R}_{K_{\infty}}$ construit sur les normes cyclotomiques.

En particulier (cf. $[\mathrm{Ko}]$ ou $\left[\mathrm{J}_{2}\right]$, th. $8(\mathrm{ii})$ ), Ker $\psi$ contient le noyau universel :

$$
\left\{\zeta_{l^{r}} \otimes x \in \mu_{l^{\infty}} \otimes \mathcal{R}_{K_{\infty}} \mid\left\{\zeta_{l^{r}}, x\right\}=1 \text { dans } H_{2}\left(K_{\infty}\right)\right\},
$$

ce qui conduit bien à l'épimorphisme annoncé : $l_{\infty} H_{2}\left(K_{\infty}\right) \rightarrow \mathbb{T}_{l} \otimes_{\mathbb{Z}_{l}} \widetilde{\mathcal{C} l} K_{\infty}^{\text {tor }}$.

Plus précisément même, la conjecture de Gross affirmant l'égalité de Ker $\psi$ et du noyau universel ainsi que celle de $\widetilde{\mathcal{C l}}_{K_{\infty}}$ et de son sous-groupe de torsion, nous obtenons dans ce cas un isomorphisme canonique

$$
l_{l} H_{2}\left(K_{\infty}\right) \simeq \mathbb{T}_{l} \otimes \widetilde{\mathcal{C l}}_{K_{\infty}},
$$

ce qui ramène l'étude arithmétique des noyaux hilbertiens attachés aux corps surcirculaires à celle des $l$-groupes de classes logarithmiques de ces corps. Comme expliqué dans $\left[\mathrm{J}_{2}\right]$, cet isomorphisme ne se redescend pas, en général, à un niveau fini de la tour cyclotomique.

\section{Références}

[CK] A. Candiotti and K. Kramer, On the 2-Sylow subgroup of the Hilbert kernel of $K_{2}$ of number fields, Acta Arith. 52 (1989), 49-65. 
[Iw] K. Iwasawa, On explicit formulas for the norm residue symbol, J. Math. Soc. Japan 20 (1968), 151-165.

$\left[\mathrm{J}_{0}\right] \quad$ J.-F. Jaulent, L'arithmétique des l-extensions, Thèse d'état, Publ. Math. Fac. Sci. Besançon, Théor. Nombres 1984-85 \& 1985/86, fasc. 1 (1986), 1-349.

$\left[\mathrm{J}_{1}\right] \quad$ - La théorie de Kummer et le $K_{2}$ des corps de nombres, Sém. Théorie des Nombres Bordeaux 2 (1990), 377-411.

$\left[\mathrm{J}_{2}\right] \quad$ - Noyau universel et valeurs absolues, Journées Arithmétiques de MarseilleLuminy, Astérisque 198-199-200 (1991), 187-207.

$\left[\mathrm{J}_{3}\right] \quad$ - Classes logarithmiques des corps de nombres, Sém. Théorie des Nombres Bordeaux, à paraître.

[Ke] F. Keune, On the structure of the $K_{2}$ of the ring of integers in a number field, K-Theory 2 (1989), 625-645.

[Ko] M. Kolster, An idelic approach to the wild kernel, Invent. Math. 103 (1991), 9-24.

[La] S. Lang, Cyclotomic Fields, Graduate Texts in Math. 59, Springer, New York, 1978.

[Se] J.-P. Serre, Corps locaux, Hermann, Paris, 1968.

[Ta] J. Tate, Relations between $K_{2}$ and Galois cohomology, Invent. Math. 36 (1976), $257-274$.

CENTRE DE RECHERCHE EN MATHÉMATIQUES DE BORDEAUX

UNIVERSITÉ BORDEAUX I

351, COURS DE LA LIBÉRATION

33405 TALENCE CEDEX, FRANCE

Reçu le 26.6.1993

et révisé le 24.1.1994 